\title{
MODELOS ANIMALES DE RETINOPATÍA DIABÉTICA: ¿DE QUÉ FORMA NOS AYUDAN?
}

\author{
ANIMAL MODELS OF DIABETIC RETINOPATHY: \\ HOW ARE THEY HELPING US?
}

\author{
UGARTE $M^{1}$
}

La retinopatía diabética $(\mathrm{RD})$ es la mayor causa de ceguera entre individuos en edad laboral en países desarrollados y la prevalencia global se estima que excederá 200 millones para el 2012. El control intensivo de la glicemia y de la tensión arterial puede reducir la incidencia de la RD. La destrucción térmica con láser de la retina puede prevenir la progresión y pérdida visual, sin embargo está asociada con un gran porcentaje de complicaciones y limitada eficacia. Es, por consiguiente, vital investigar nuevas terapias con mecanismos de acción novedosos. Un mayor entendimiento de los mecanismos bioquímicos involucrados en el inicio y progreso de la RD es esencial para el desarrollo de nuevos agentes terapéuticos y el uso de modelos animales proporciona la vía para ello, dado que la fisiología del ratón, la rata y otros animales (por ejemplo, el mono) pueden ser muy similares a la condición humana.

Se sabe que la glicemia crónica en diabetes es la mayor causa de daño a los vasos sanguíneos, neuronas y células gliales de la retina. Sin embargo, la complejidad para crear un verdadero modelo representativo de la RD humana va más allá del desarrollo de métodos para elevar los niveles de azúcar en el suero de un animal en concreto. Ninguno de los modelos animales disponibles actualmente representa todas las características de la RD. Cada modelo reproduce un aspecto relevante de la enfermedad a la vez que incluye características fisiológicas únicas que influyen la interpretación de los resultados experimentales. Las moléculas pueden tener funciones muy diferentes en animales de laboratorio, y pueden ser expresadas en diferentes tipos celulares. Estas características pueden ser únicas a una especie animal de línea genética. Otra deficiencia inherente a los modelos animales es la especificad de cada caso. La mayoría de las líneas animales utilizadas en investigación médica son engendrados por endogamia. Pacientes humanos, por el contrario, son genéticamente heterogeneos. El origen de su enfermedad, al igual que sus respuestas al tratamiento, pueden variar enormemente. Una línea específica de ratón o rata puede considerarse que representa, en el mayor de los casos, un único subgrupo de pacientes humanos. La validación de terapias en más de un modelo animal debería resolver muchos de los problemas específicos a una especie y línea. Un fármaco que tiene efecto en ratas y ratones transgénicos tiene más probabilidades de modificar un mecanismo universal de la enfermedad, el cual es más probable que también este presente en humanos.

La mayoría de los modelos de RD imitan diabetes tipo 1, causada por destrucción de las células beta de los islotes pancreáticos. Los ratones y las ratas inyectados con estreptozotocina (STZ) o alloxan pierden las células beta y se convierten en dependientes a la insulina. Este enfoque replica algunos de los síntomas tempranos de la RD y tiene la ventaja de que el comienzo de la diabetes puede definirse cómo el momento de la inyección de la toxina. Se han reconocido muchas variaciones entre especies y dentro de la misma especie, tanto en la bioquímica retiniana como en las respuestas histopatológicas a «insultos» diabéticos idénticos. Las ratas inyectadas con STZ pierden los pericitos y capilares retinianos, desarrollan engrosamiento de la membrana basal vascular y un aumento de la permeabilidad vascular. En las ratas inyectadas con STZ, los cambios fisiológicos y bioquímicos en la retina aparecen 1-2 meses después de la hiperglicemia. Las células de Müller muestran cambios tras 2-5 meses de diabetes experimental. La obliteración capilar es evidente por

\footnotetext{
${ }^{1}$ NIHR Clinical Lecturer in Ophthalmology, Manchester Academic Health Sciencies Centre and NIHR Manchester Biomedical Research Centre, University of Manchester, UK.

E-mail: marta.ugarte@manchester.ac.uk
} 
primera vez después de seis meses de diabetes. Las abnormalidades no vasculares (neuronales y gliales) inducidas por la diabetes preceden al desarrollo de cambios en células vasculares en las ratas y es posible que contribuyan en la patogenicidad de la enfermedad vascular en este modelo (1).

El poder de los modelos de ratón en la RD reside en la facilidad con la que se pueden introducir cambios genéticos para explicar los mecanismos moleculares involucrados en el inicio y la progresión de la enfermedad. Sin embargo, la diabetes inducida por STZ en ratón tiene menos éxito que en la rata, debido a la resistencia del ratón a la toxina. Los ratones inyectados con STZ desarrollan alteraciones vasculares características de la RD temprana en la rata, en concreto la inducción de capilares acelulares, apoptosis de células vasculares y la formación de pericitos «fantasma», 6 meses tras la inducción de la diabetes, a pesar de la falta de pérdida neuronal significativa y activación glial persistente (2). Esto sugiere que los procesos que dan lugar a los cambios vasculares son de alguna forma diferentes a los que desencadenan cambios no vasculares en el ratón, permitiendo así el estudio de los cambios vasculares de forma aislada.

También existen modelos que desarrollan diabetes tipo 1 de forma espontánea, por ejemplo el modelo de ratón Ins2Akita (3), el cual posee una mutación dominante en el gen de la insulina 2. Esto da lugar al mal repliege de la insulina, la muerte de las células beta pancreáticas, hipoinsulinemia sistémica e hiperglicemia, a las 4 semanas de edad. Las retinas de los machos heterozigóticos muestran abnormalidades vasculares, neurales y gliales similares a los cambios detectados en las ratas inyectadas con STZ, pero el uso del ratón Ins2Akita tiene varias ventajas. Primero, la mutación dominante permite el estudio de animales heterozigóticos. Segundo, los ratones son fértiles y se reproducen mejor que las ratas. Tercero, tienen diabetes con deficiencia de insulina estable y pueden ser mantenidos en un estado menos catabólico sin insulina exógena. Cuarto, el mecanismo de inicio de la diabetes no involucra alteraciones inmunológicas.

La «verdadera» neovascularización preretiniana solo se ha demostrado en perros alimentados con elevadas cantidades de galactosa (4), un modelo con niveles elevados de azúcar en plasma y vida prolongada, permitiendo el desarrollo de cambios vasculares en la retina tanto con estados iniciales como tardíos de la retinopatía.
Hay relativamente pocos estudios comparando el desarrollo de la retinopatía en diabetes tipo 1 y 2 . Sin embargo, es probable que haya pequeñas diferencias entre ellas. Solo hay unos pocos modelos de verdadera diabetes tipo 2, la cual está asociada con obesidad y resistencia a la insulina. Las ratas obesas Zucker obese, la rata gorda diabética Tori y la rata gorda Otsuka Long-Evans Tokushima son ejemplos de ratas que desarrollan diabetes tipo 2 de forma espontánea. Los ratones Ob/ob 9 y KKAY son buenos modelos de algunos cambios metabólicos de la diabetes tipo 2. Sin embargo, ni las ratas, ni los ratones parecen desarrollar cambios en la retina similares a la retinopatía diabética en humanos con diabetes tipo 2 .

El único modelo animal disponible actualmente que demuestra manifestaciones de diabetes similares a los humanos es un mono con prolongada diabetes tipo 2, el cual desarrolla pérdida de capilares, hemorragias intraretinianas y microaneurismas (5). A pesar del alto coste y del consumo de tiempo, especies de animales grandes merecen ser considerados por su posible papel como «modelos funcionales». Representan pre-condicionamiento fisiológico y natural único al igual que ventajas experimentals de gran valor en el desarrollo de modelos alternativos de RD.

A pesar de que la mayoría de la investigación de enfermedades es llevada a cabo en vivo, Huub Kreuwel de Entelos y Mark Atkinson de la Universidad de Florida han desarrollado un modelo virtual uniendo los órganos mayores de un modelo de ratón virtual. Este ratón consiste de módulos interconectados representando células, tejidos y órganos. Algoritmos matemáticos de cada tejido conforman el conjunto de parámetros determinados en previos estudios de ratón. La ventajas únicas potenciales de este modelo incluyen la posibilidad de seguir niveles continuos de células reguladoras, insulina, y otras biomoléculas, determinando la dosis optima del fármaco, e incluso reconciliando resultados de estudios aparentemente conflictivos. Las alteraciones del programa permitirían la representación de líneas de ratón adicionales y en el futuro podría ser utilizado en la investigación de la RD.

Dado que actualmente no existe un único modelo que reproduzca de forma completa los mecanismos involucrados en la RD en humanos, cada investigador debe elegir los modelos que reproducen un aspecto relevante de la enfermedad en concreto.

Dados los formidables obstáculos que existen en el desarrollo de modelos de la situación humana, no 
es de extrañar que la correlación entre estudios con modelos animales y pacientes humanos no haya sido perfecta. El tratamiento con los inhibidores de la proteina kinasa $\mathrm{C}(\mathrm{PKC})$ es un ejemplo relevante. Se demostró que protegía relativa o completamente el edema macular en modelos animales de RD. Sin embargo, en los 10 últimos años, se ha ido esclareciendo que los inhibidores de la PKC tienen poco o ningún efecto protector en humanos. La ausencia de acuerdo en el efecto de estos agentes en animales y humanos puede ser debido a artefactos de los modelos animales, diferencias en la fisiología y efecto terapeútico entre los animales de laboratorio y los humanos, y/o las discrepancias entre el «estado equivalente de la enfermedad» en animales y humanos. El comienzo de la RD en humanos se define con el desarrollo de cambios vasculares identificados clínicamente. En animales, la «retinopatía» inducida por la diabetes se considera que comienza mucho antes, cuando los cambios en los parámetros de laboratorio tienen lugar. Se sabe que el momento del inicio de la terapia es crítico para revertir los cambios inducidos por la diabetes en animales de laboratorio. Esto sugiere que algunas terapias han podido fallar en humanos porque el tratamiento comenzó en «mal» momento.

El camino desde las pruebas animales hasta el desarrollo de terapias para la RD en humanos ha sido muy difícil hasta el momento. Sin embargo, el uso de animales continuará contribuyendo de forma substancial al estudio de mecanismos moleculares de la patofisiología de la RD. La consideración de las diferencias entre animales y humanos debería ayudar a facilitar el camino al descubrimiento de tratamientos con efecto beneficioso en la clínica (¡en un futuro próximo!).

\section{BIBLIOGRAFÍA}

1. Karachalias N, Babaei-Jadidi R, Ahmed N, Thornalley PJ. Accumulation of fructosyl-lysine and advanced glycation end products in the kidney, retina and peripheral nerve of streptozotocin-induced diabetic rats. Biochem Soc Trans 2003; 3: 1423-1425.

2. Feit-Leichman RA, Kinouchi R, Takeda M, Fan Z, Mohr S, Kern TS, et al. Vascular damage in a mouse model of diabetic retinopathy: relation to neuronal and glial changes. Invest Ophthalmol Vis Sci 2005; 46: 4281-4287.

3. Barber AJ, Antonetti DA, Kern TS, Reiter CE, Soans RS, Krady JK, et al. The Ins2Akita mouse as a model of early retinal complications in diabetes. Invest Ophthalmol Vis Sci 2005; 46: 2210-2218.

4. Kador PF, Takahashi Y, Wyman M, Ferris F 3rd. Diabetes like proliferative retinal changes in galactose-fed dogs. Arch Ophthalmol 1995; 113: 352-354.

5. Kim SY, Johnson MA, McLeod DS, Alexander T, Otsuji T, Steidl SM, et al. Retinopathy in monkeys with spontaneous type 2 diabetes. Invest Ophthalmol Vis Sci 2004; 45: 4543-4553. 